\title{
Effect of in-material losses on terahertz absorption, transmission, and reflection in photonic crystals made of polar dielectrics
}

\author{
Andriy E. Serebryannikov, S. Nojima, K. B. Alici, and Ekmel Ozbay
}

Citation: Journal of Applied Physics 118, 133101 (2015);

View online: https://doi.org/10.1063/1.4932017

View Table of Contents: http://aip.scitation.org/toc/jap/118/13

Published by the American Institute of Physics

\section{Articles you may be interested in}

Enhanced transmission and beaming via a zero-index photonic crystal

Applied Physics Letters 109, 031105 (2016); 10.1063/1.4959085

Diffraction inspired unidirectional and bidirectional beam splitting in defect-containing photonic structures without interface corrugations

Journal of Applied Physics 119, 193108 (2016); 10.1063/1.4949509

Experimental demonstration of deflection angle tuning in unidirectional fishnet metamaterials at millimeter-waves Applied Physics Letters 106, 061109 (2015); 10.1063/1.4908260

Propagation of acoustic waves in a metamaterial with a refractive index of near zero Applied Physics Letters 102, 241906 (2013); 10.1063/1.4811742

Uniaxial epsilon-near-zero metamaterial for angular filtering and polarization control Applied Physics Letters 97, 131107 (2010); 10.1063/1.3469925

Broadband metasurfaces enabling arbitrarily large delay-bandwidth products Applied Physics Letters 108, 031601 (2016); 10.1063/1.4939979

\section{Scilight} Sharp, quick summaries illuminating the latest physics research

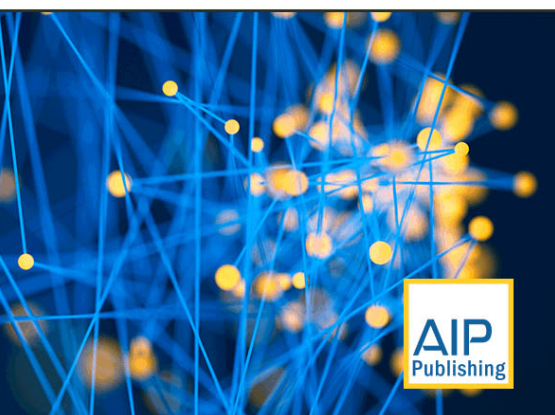




\title{
Effect of in-material losses on terahertz absorption, transmission, and reflection in photonic crystals made of polar dielectrics
}

\author{
Andriy E. Serebryannikov, ${ }^{1,2, a)}$ S. Nojima, ${ }^{3}$ K. B. Alici, ${ }^{4}$ and Ekmel Ozbay ${ }^{2}$ \\ ${ }^{1}$ Faculty of Physics, Adam Mickiewicz University, 61-614 Poznań, Poland \\ ${ }^{2}$ Nanotechnology Research Center-NANOTAM, Bilkent University, 06800 Ankara, Turkey \\ ${ }^{3}$ Yokohama City University, Department of Nanosystem Science, Graduate School of Nanobioscience, \\ Kanazawa Ku, 22-2 Seto, Yokohama, Kanagawa 2360027, Japan \\ ${ }^{4}$ TUBITAK Marmara Research Center, Materials Institute, 41470 Gebze, Kocaeli, Turkey
}

(Received 24 August 2015; accepted 9 September 2015; published online 1 October 2015)

\begin{abstract}
The effect of the material absorption factor on terahertz absorption $(A)$, transmittance $(T)$, and reflectance $(R)$ for slabs of $\mathrm{PhC}$ that comprise rods made of GaAs, a polar dielectric, is studied. The main goal was to illustrate how critical a choice of the absorption factor for simulations is and to indicate the importance of the possible modification of the absorption ability by using either active or lossy impurities. The spectra of $A, T$, and $R$ are strongly sensitive to the location of the polaritonic gap with respect to the photonic pass and stop bands connected with periodicity that enables the efficient combination of the effects of material and structural parameters. It will be shown that the spectra can strongly depend on the utilized value of the material absorption factor. In particular, both narrow and wide absorption bands may appear owing to a variation of the material parameters with a frequency in the vicinity of the polaritonic gap. The latter are often achieved at wideband suppression of transmission, so that an ultra-wide stop band can appear as a result of adjustment of the stop bands having different origin. The results obtained at simultaneous variation of the absorption factor and frequency, and angle of incidence and frequency, indicate the possibility of the existence of wide ranges of tolerance, in which the basic features do remain. This allows for mitigating the accuracy requirements for the absorption factor in simulations and promises the efficient absorption of nonmonochromatic waves and beams with a wide angular spectrum. Suppression of narrowband effects in transmission is demonstrated at rather large values of the absorption factor, when they appear due to either the defect modes related to structural defects or dispersion inspired variations of the material parameters in the vicinity of the polaritonic gap. Comparison with auxiliary structures helps one to detect the common features and differences of homogeneous slabs and slabs of a PhC, which are made of GaAs. C 2015 AIP Publishing LLC.
\end{abstract}

[http://dx.doi.org/10.1063/1.4932017]

\section{INTRODUCTION}

Polar dielectrics like $\mathrm{GaAs}, \mathrm{LiF}$, and $\mathrm{NaCl}$ are considered as perspective materials for various terahertz applications. They show strong dispersion and quite strong absorption within a frequency range, whose width and location depend on the material. The basic effects exerted by polar dielectrics are connected with the polaritonic gap, which appears owing to the coupling of photons to transverse optical phonons. ${ }^{1}$ In particular, the ranges of high and near-zero permittivity can be useful. Similarly to the other mechanisms leading to narrowband effects, such as slow waves ${ }^{2}$ and defect modes arising due to structural defects, ${ }^{3}$ narrowband variations of material parameters can lead to strong narrowband variations in transmittance $(T)$, reflectance $(R)$, and absorption $(A){ }^{4}$

New features can appear in the structures with the periodic arrangement of multiple individual components made of polar dielectrics. For instance, multiple excitonic polaritons and multiple gaps have been demonstrated in onedimensional photonic crystals (PhCs) containing polar

${ }^{\text {a)} E l e c t r o n i c ~ m a i l: ~ a n d s e r @ a m u . e d u . p l . ~}$ dielectrics. $^{5-7}$ Rod-type metamaterials enabling hyperbolic dispersion, superlensing, near-zero, and negative permeability have been proposed., Recently, various reflection-free regimes have become the focus of interest, in which either perfect transmission or perfect absorption is achieved. In this concern, one should mention perfect transmission in the deflection mode, ${ }^{10,11}$ perfect one-way absorbers with high transmission in the neighboring bands, ${ }^{12}$ and ultrathin, (nearly) perfect absorbers for various frequency ranges. ${ }^{13-22}$ In spite of the fact that many absorbers were designed for terahertz frequencies, the potential of polar dielectrics in such devices has not yet been fully exploited. Clearly, absorption regimes achievable in more or less complex structures depend on the properties of the lossy materials that are comprised by these structures. Thus, the knowledge of the actual losses for the utilized material and a correct setting of the absorption factor in simulations of the corresponding theoretical performances are very important. On the other hand, using active or lossy impurities, correspondingly, for reducing or enhancing the resulting losses looks perspective. In fact, searching for better, i.e., more suitable materials becomes an important trend. As an example, a study dedicated to the search for plasmonic materials with reduced losses should be mentioned. ${ }^{23}$ 
In the present paper, we study the effect exerted by variations of the absorption factor of GaAs on the spectra of $A$, $T$, and $R$ for a $\mathrm{PhC}$ composed of the rods made of this material. Different possible locations of the polaritonic gap with respect to the photonic pass and stop bands will be considered, while the main attention is paid to the case of the polaritonic gap being located at the upper edge of the lowest stop band. It will be shown that not only absorption but also transmission and reflection spectra can be changed dramatically, for instance, at a tenfold variation of the absorption factor. We clarify which features are inherited from homogeneous slabs of GaAs and which ones from the slab of $\mathrm{PhC}$ in zero-loss approximation. Then, absorption will be studied for PhCs at simultaneous variation of (i) frequency and the material absorption factor when angle of incidence is fixed and (ii) frequency and angle when the absorption factor is fixed. The emphasis will be put on the investigation of whether the basic features in the behavior of $A$ can be preserved for rather wide ranges of variation of the abovementioned parameters, at least in the extent sufficient for broadband operation and obtaining of tolerance with respect to the settings used for frequency, angle, and absorption factor. Thereafter, the possibility of suppression of the effect of defect modes in transmission will briefly be discussed. Finally, the specifics of subwavelength regime will be considered for both slabs of a PhC and homogeneous slabs of GaAs. The presented results are obtained by using the coupled integral equation technique. ${ }^{24}$

\section{THEORETICAL BACKGROUND}

Phonon-photon coupling and polariton excitation occur in polar dielectrics leading to strong frequency dispersion. The permittivity of polar dielectrics is usually described as follows: ${ }^{1,25}$

$$
\varepsilon_{P}(\omega)=\varepsilon_{\infty}+\left(\varepsilon_{0}-\varepsilon_{\infty}\right) \omega_{T}^{2} /\left(\omega_{T}^{2}-\omega^{2}-i \Gamma \omega\right),
$$

where $\varepsilon_{0}$ is the static permittivity, $\varepsilon_{\infty}$ is the high-frequency permittivity, and $\Gamma$ is the absorption factor.

In lossless case, the range of $\operatorname{Re} \varepsilon_{P}<0$ corresponds to the polaritonic gap, with the lower and upper boundaries that are denoted by the angular frequencies $\omega_{T}$ and $\omega_{L}$, respectively. Throughout the paper, location of the polaritonic gap is schematically shown at top of the plots by a rectangle. $\omega_{T}$ and $\omega_{L}$ are connected by the Lyddane-SachsTeller relation ${ }^{1}$

$$
\omega_{L}^{2} / \omega_{T}^{2}=\varepsilon_{0} / \varepsilon_{\infty}
$$

The actual frequency range, in which $\operatorname{Re} \varepsilon_{P}<0$, is always narrower than the range of $\omega_{T} / 2 \pi<f<\omega_{L} / 2 \pi$ because $\Gamma \neq 0$. In this paper, consideration is restricted to GaAs, which is probably one of the most widely used polar dielectrics. For this material, $\omega_{T} /(2 \pi)=8.12 \mathrm{THz}$ and $\omega_{L} /(2 \pi)=8.75 \mathrm{THz} .{ }^{1,25}$ Examples of the frequency dependencies of $\operatorname{Re} \varepsilon_{P}$ and $\operatorname{Im} \varepsilon_{P}$ are presented in Fig. 1. The smaller $\Gamma / \omega_{T}$, the stronger resonance behavior is pronounced and the larger $\operatorname{Re} \varepsilon_{P}$ and $\operatorname{Im} \varepsilon_{P}$ can be. It is known that $A \propto \operatorname{Im} \varepsilon_{P} \int_{V} E^{2} d V$, where $E$ is electric field and $V$ is volume occupied by the absorbing material. Thus,
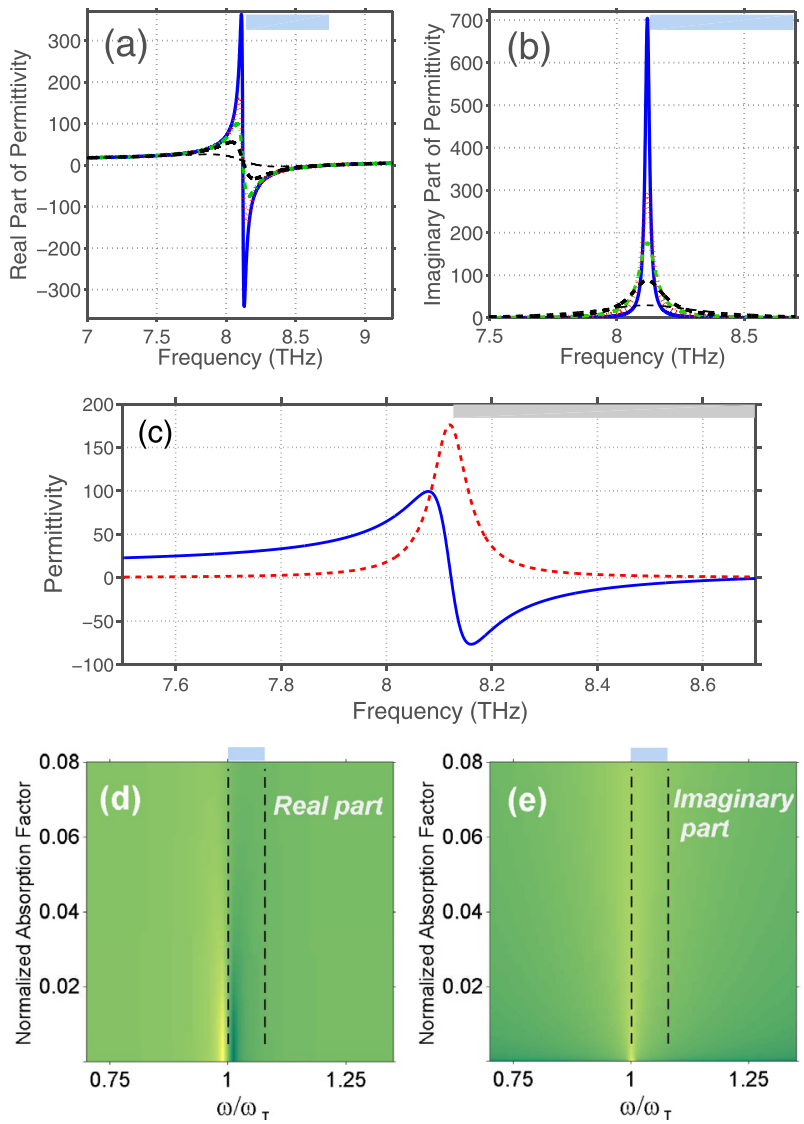

FIG. 1. (a) $\operatorname{Re} \varepsilon_{P}$ and (b) $\operatorname{Im} \varepsilon_{P}$ vs frequency for GaAs at different values of $\Gamma / \omega_{T}$ : solid blue line $-2.5 \times 10^{-3}$, dotted red line $-6 \times 10^{-3}$, dasheddotted green line $-10^{-2}$, thick dashed black line $-2 \times 10^{-2}$, thin dashed black line $-6 \times 10^{-2}$; (c) $\operatorname{Re} \varepsilon_{P}$ (solid blue line) and $\operatorname{Im} \varepsilon_{P}$ (dashed red line) in one plot for demonstration of typical location of the maxima, $\Gamma / \omega_{T}=10^{-2}$; (d) $\operatorname{Re} \varepsilon_{P}$ and (e) $\lg \left(\operatorname{Im} \varepsilon_{P}\right)$ for GaAs in $\left(\omega / \omega_{T}, \Gamma / \omega_{T}\right)$-plane, location of polaritonic gap is shown also by vertical dashed lines.

absorption can be strong also at very small values of $\operatorname{Im} \varepsilon_{P}$ provided that $E$ is strong enough. In turn, the last condition needs such material parameters that the incident field is not fully reflected at the material boundaries but can penetrate into it. As known from the earlier studies, $\max A$ can be achieved at the values of $\operatorname{Re} \varepsilon_{P}$ and $\operatorname{Im} \varepsilon_{P}$ that are (much) smaller than the maximal ones, ${ }^{12}$ if the resulting mechanism is based on interferences. ${ }^{21,26,27}$ Hence, both the correct choice of $\Gamma / \omega_{T}$ for an existing material sample and a route to the efficient engineering of the materials with values of $\Gamma / \omega_{T}$, which would enable a desired behavior of $\operatorname{Re} \varepsilon_{P}$ and $\operatorname{Im} \varepsilon_{P}$ and, thus, of $T, R$, and $A$, should be very important for the structures containing polar dielectrics. It is noteworthy that the frequency dependent term in Eq. (1) does not tend to zero when $1-\omega / \omega_{T}$ and $\Gamma$ are simultaneously decreased. $\varepsilon_{P}$ remains finite for all the values of $\omega$ if $\Gamma>0$.

The general geometry of the problem is presented in Fig. 2(a). An s-polarized electromagnetic wave is incident on a slab of the rod-type $\mathrm{PhC}$ at the angle $\theta$. The structure is assumed to be infinitely extended in the $x$-direction. The electric field vector of the incident wave is directed along the rod axes. The circular rods of diameter $d$ are located in square lattice with the constant $a$, whereas the virtual 


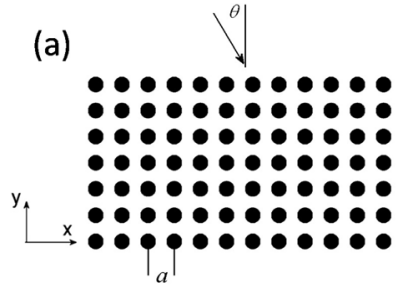

(c)
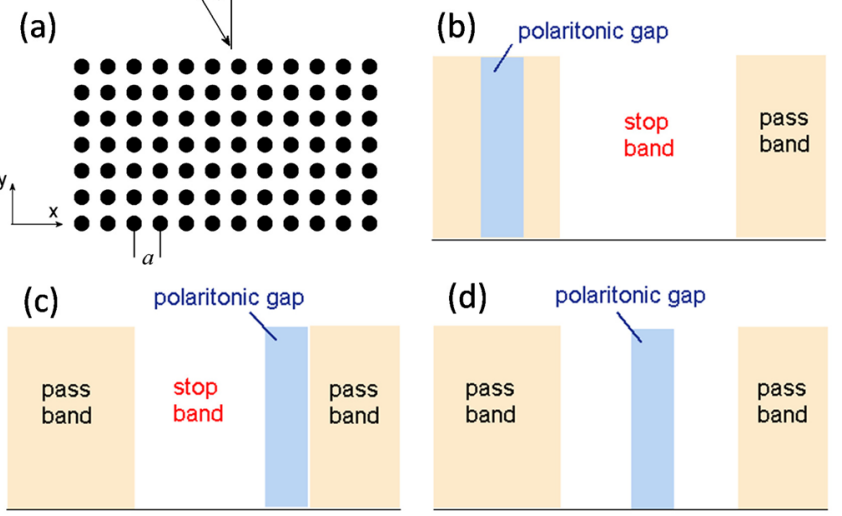

FIG. 2. (a) General geometry of the problem and (b, c, d) possible cases of location of polaritonic gap with respect to pass and stop bands arising due to the periodicity: (b) inside the lowest pass band, (c) at the upper edge of the lowest stop band, and (d) in the middle of the lowest stop band.

interfaces are along $\Gamma-\mathrm{X}$ direction. The slab contains $N$ layers of the rods.

Figures 2(b)-2(d) schematically show three possible cases of location of the polaritonic gap of GaAs with respect to the lowest pass and stop bands arising due to the periodicity in the similar $\mathrm{PhC}$ based structure with the rods having $\varepsilon=\varepsilon_{\infty}$. For the first of them, the polaritonic gap is located inside the lowest pass band, i.e., at lower frequencies than the lowest stop band. For the second case, it is located inside the lowest stop band, being adjacent to its upper edge. For the third one, it is located in the middle of the stop band. Hence, different spectral features of $T, R$, and $A$ can be obtained just by varying $a$ and $d$, because $\omega_{T}$ and $\omega_{L}$ are fixed. The case in Fig. 2(d) has been utilized in one-way absorbers containing rod layers. ${ }^{12}$ The case in Fig. 2(b) might be close to the polar dielectric based metamaterials. ${ }^{8}$ In this paper, more attention will be paid to the case being similar to that in Fig. 2(c).

The values of $T$ and $R$ are obtained at given frequency, material, and geometrical parameters from iterative solution of the coupled integral equations in the spectral domain. ${ }^{24}$ Then, absorption is calculated from the energy balance condition, i.e., $A=1-T-R$. The rich experience of using the integral equation technique ${ }^{24}$ in solution of various problems for PhCs and other periodic structures indicates the possibility of obtaining the appropriate convergence at reasonable computational costs. In order to better connect with the periodicity relevant features, we use-instead of frequencydimensionless units that are conventional for $\mathrm{PhCs}$, i.e., $k a=2 \pi f a / c$, where $c$ is velocity of electromagnetic wave in vacuum. In fact, this means that the theoretical performances corresponding to different locations of the polaritonic gap and, thus, to different values of $k_{T} a\left(k_{T}=\omega_{T} / c\right)$ are distinguished in value of $a$, while $a / d$ is kept constant. These performances are not re-scalable in sense that the value of $a$ is unambiguously set for any fixed value of $k_{T} a$. For example, $k_{T} a=7 \pi / 12$ corresponds to $a=10.8 \mu \mathrm{m}$.

\section{BASIC EFFECTS OF $\Gamma$}

First, let us consider the effects exerted by variations in $\Gamma$ on the transmittance, reflectance, and absorption of the $\mathrm{PhC}$ comprising eight layers of GaAs rods. Some effects have been studied for similar structures. ${ }^{25,28,29}$ The aim here is to demonstrate (i) how important a choice of the value of $\Gamma$ is and (ii) the role of the losses in obtaining desired features of the spectra of $T, R$, and $A$. A typical scenario of evolution of the spectra at varying $\Gamma$ from small to large values and richness of the related effects will be illustrated. For the comparison, the case of the rods made of a dispersion-free material with $\varepsilon=\varepsilon_{\infty}$ is also considered. Geometric parameters are chosen such that the polaritonic gap is located similarly to Fig. 2(c). The results are presented in Fig. 3. It is seen that taking into account the frequency dependent term in Eq. (1) can result in a strong modification of the spectra-compare Figs. 3(a) and 3(b). In particular, three new pass bands appear in the vicinity of $k a=2.4, k a=2.55$, and $k a=2.8$, see Fig. 4(a) for details. At the same time, the old stop band from Fig. 3(a) is narrowing. Locations of the old and new stop bands could approximately be described by the qualitative theory suggested in Ref. 25. Strong absorption is observed in Figs. 3(b) and 4(a) only between $k a=2.55$ and $k a=2.62$, while the polaritonic band extends for the chosen value of $k_{T} a$ from $k a=2.62$ to $k a=2.82$. It is noteworthy that the third new pass band centered at $k a=2.8$ partially coincides with the polaritonic gap.
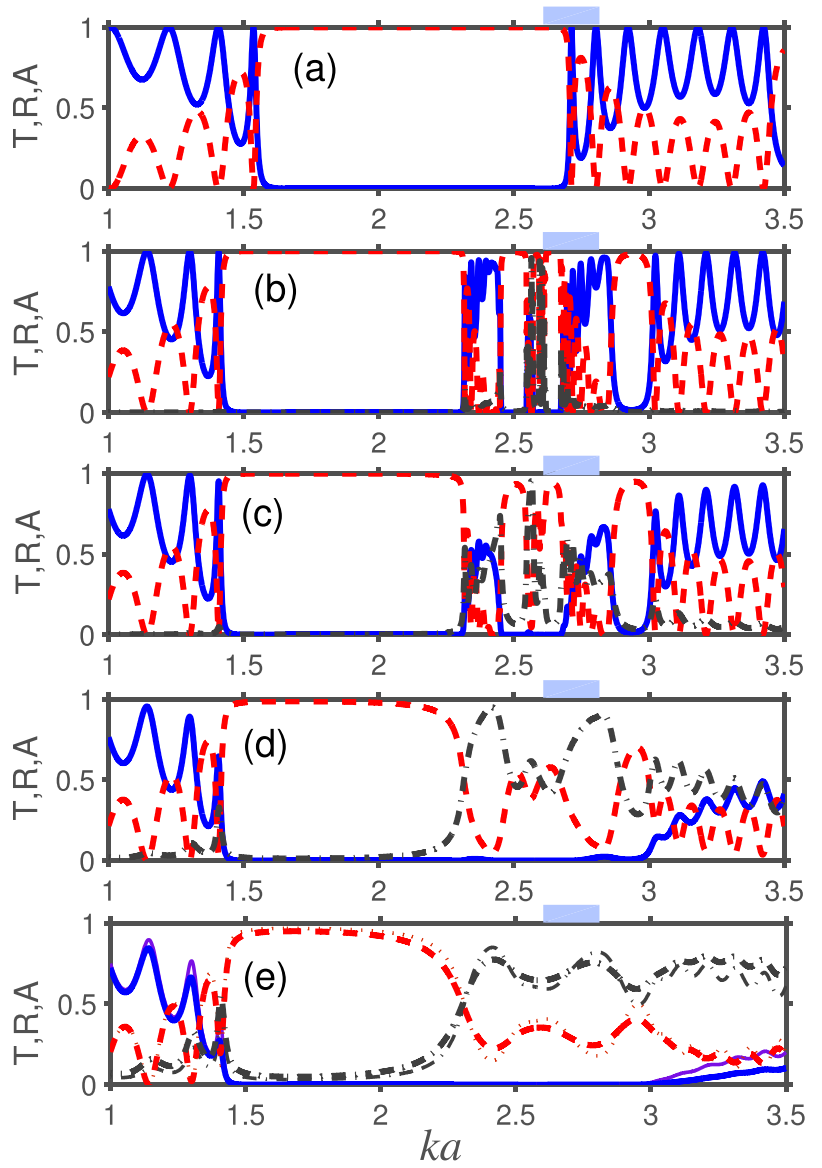

FIG. 3. Transmittance (solid blue lines), reflectance (dashed red lines), and absorption (dashed-dotted black lines) for slab of PhC at (a) $\varepsilon_{P}=\varepsilon_{\infty}=10.9$ and (b)-(e) $\varepsilon_{P}$ given by (1) with $\varepsilon_{\infty}=10.9, \quad \varepsilon_{0}=12.66$, (b) $\Gamma / \omega_{T}$ $=2.5 \times 10^{-4}$, (c) $\Gamma / \omega_{T}=2.5 \times 10^{-3}$, (d) $\Gamma / \omega_{T}=2.5 \times 10^{-2}$, and (e) $\Gamma / \omega_{T}=6 \times 10^{-2}$ (thick lines) and $9.5 \times 10^{-2}$ (thin lines); $k_{T} a=5 \pi / 6$, $d / a=0.4, N=8$, and $\theta=0$. 

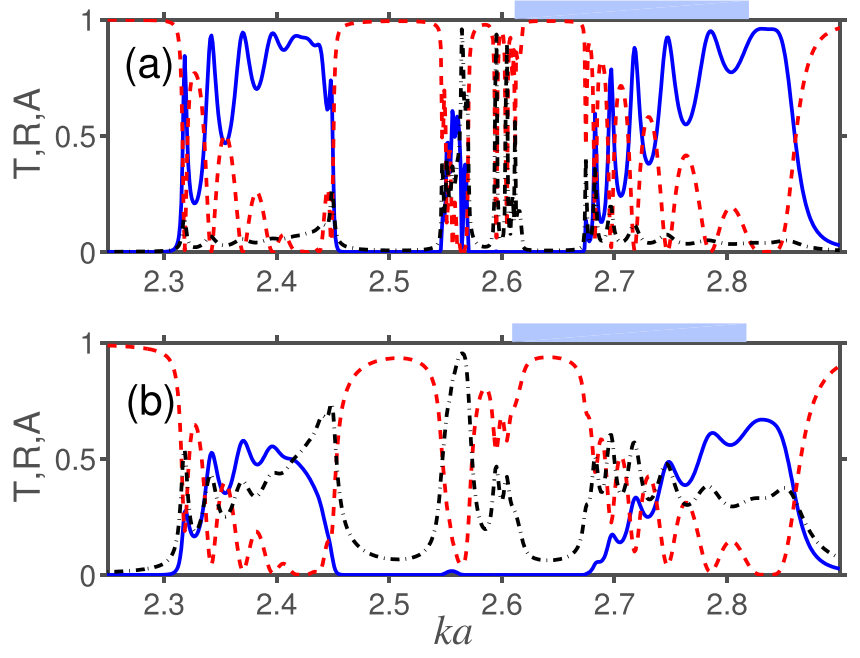

FIG. 4. Enlarged fragments of (a) Fig. 3(b) and (b) Fig. 3(c).

Thus, the studied structure emulates a slab of metallic-rod $\mathrm{PhC}$ in this case. ${ }^{30-32}$ In fact, $\operatorname{Re} \varepsilon_{P}$ varies from -29 at $k a=2.675$ to 3.2 at $k a=2.9$. Hence, the third band partially corresponds to the $\mathrm{PhC}$ made of an epsilon-near-zero material $^{33}$ and partially to that made of a conventional dielectric. On the contrary, the first and second new bands correspond to large positive values of $\operatorname{Re} \varepsilon_{P}$. In particular, $\operatorname{Re} \varepsilon_{P}=19$ at $k a=2.31$ and $\operatorname{Re} \varepsilon_{P}=25$ at $k a=2.45$ for the first band, while $\operatorname{Re} \varepsilon_{P}=43$ at $k a=2.545$ and $\operatorname{Re} \varepsilon_{P}=59$ at $k a=2.57$, see Fig. 4(a). In turn, the above-mentioned ranges of strong absorption correspond to $\operatorname{Re} \varepsilon_{P}>40$ and $\operatorname{Im} \varepsilon_{P}>0.15$, which are still below the maxima of $\operatorname{Re} \varepsilon_{P}$ and $\operatorname{Im} \varepsilon_{P}$, see Fig. 1 .

The increase of $\Gamma / \omega_{T}$ from $2.5 \times 10^{-4}$ to $2.5 \times 10^{-3}$ results in a significant increase of $A$ in the new pass bands, see Fig. 3(c). Moreover, the new pass band located at $k a=2.55$ tends to vanish. In turn, wide absorption bands appear around $k a=2.4,2.56,2.6$, and 2.75. Details are shown in Fig. 4(b). At the next tenfold increase of $\Gamma / \omega_{T}$, the spectra of $T, R$, and $A$ are dramatically changed, as seen in Fig. 3(d). The main difference between Figs. 3(c) and 3(d) is that now the new pass bands disappear, whereas an absorption band is formed, which is substantially wider than the polaritonic gap. A new wide stop band appears that is wider than that in Fig. 3(a) owing to the common effect of the photonic and polaritonic gaps. Indeed, it is seen that $R>0.95$ at $1.45<k a<2.15$, while $A>0.42$ at $2.31<k a<2.9$. Two maxima of $A$ observed in Fig. 3(d) are located at the different sides with respect to the polaritonic gap. In other words, the first of them should correspond to large positive values of $\operatorname{Re} \varepsilon_{P}$, while the second one does to $0<\operatorname{Re} \varepsilon_{P}<1$. Accordingly, the dominant mechanisms of absorption at these maxima might be different. Note that the maxima of $A$ do not coincide with those of $\operatorname{Re} \varepsilon_{P}$ and $\operatorname{Im} \varepsilon_{P}$. The further increase of $\Gamma / \omega_{T}$, i.e., from $2.5 \times 10^{-2}$ to $6 \times$ $10^{-2}$ and then to $9.5 \times 10^{-2}$, leads even to a wider absorption band. Now, it includes the range of $3<k a<3.5$, in which the constructive interference is finally destroyed, compare Figs. 3(d) and 3(e). The absorption band in Fig. 3(e) corresponds to a wide range of variation of $\operatorname{Re} \varepsilon_{P}$, which includes highepsilon, epsilon-negative, epsilon-near-zero, and conventional dielectric subranges. Besides, a well pronounced switching between the ranges of dominant reflection and absorption is observed here at $k a=2.3$. To summarize, the strong effect of $\Gamma / \omega_{T}$ on the spectra of $T, R$, and $A$ is evident.

More scenarios can be realized due to a proper choice of $k_{T} a$ and $N$. Two examples are presented in Fig. 5. In Fig. 5(a), the case of $A \approx 1$ is obtained in the middle of the lowest photonic stop band, where the polaritonic gap is located according to Fig. 2(d). It is interesting that $\max A$ is achieved here at the upper edge of the polaritonic gap [similarly to one of the maxima of $A$ in Fig. 3(d)], where $\operatorname{Re} \varepsilon_{P}>1$ and $\operatorname{Im} \varepsilon_{P}>1$ at the chosen values of $k_{T} a$ and $\Gamma / \omega_{T}$. In Fig. 5(b), even a wider stop band and sharper switching between the regimes of strong reflection and strong absorption are obtained, as compared to Fig. 3(e), at the price of increase of $k_{T} a$ and $N$. The latter occurs near $k a=2.4$. In contrast with Figs. 2(b)-2(d) and 5(a), the polaritonic gap is located here inside the second lowest pass band of the original dispersionfree structure in Fig. 3(a) but rather close to its lower edge. Similarly to Fig. 3(e), a wide absorption band includes the parts corresponding to very different ranges of variation of $\operatorname{Re} \varepsilon_{P}$ and $\operatorname{Im} \varepsilon_{P}$. Hence, the common effect of variation of $\Gamma / \omega_{T}, k_{T} a$, and $N$ in the studied periodic structures can manifest itself in a rich variety of the interesting features in the spectra of $T, R$, and $A$.

\section{AUXILIARY STRUCTURES AND INHERITING}

Now, let us try to clarify which of the features observed in Figs. 3 and 4 are mainly connected with the effect of $\operatorname{Re} \varepsilon_{P}$ and which ones with the effect of $\operatorname{Im} \varepsilon_{P}$. Figure 6 presents the results for two selected values of $\Gamma / \omega_{T}$. The only difference compared to Figs. 3(c) and 3(e) is that now $\operatorname{Im} \varepsilon_{P}=0$. The dependencies of $T$ and $R$ on $k a$ in Fig. 6(a) remain nearly the same as in Fig. 3(c), at least for $k a<2.5$ and $2.8<k a<3.5$. There are several mini pass bands (maxima of $T$ ) around $k a=2.55$ (not well seen), which are replaced with the
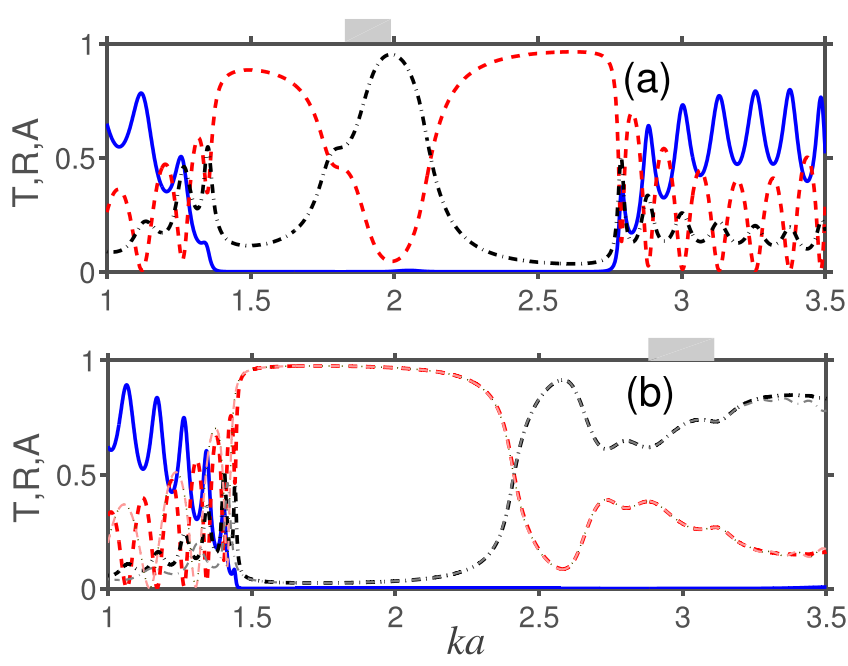

FIG. 5. Transmittance (solid blue lines), reflectance (dashed red lines), and absorption (dashed-dotted black lines) for slab of $\mathrm{PhC}$ with the rods having $\varepsilon=\varepsilon_{P}\left[\right.$ Eq. (1)] at $\varepsilon_{\infty}=10.9, \varepsilon_{0}=12.66, d / a=0.4, \Gamma / \omega_{T}=6 \times 10^{-2}$, (a) $k_{T} a=7 \pi / 12, N=8$, and (b) $k_{T} a=11 \pi / 12, N=14 ; \theta=0$. 

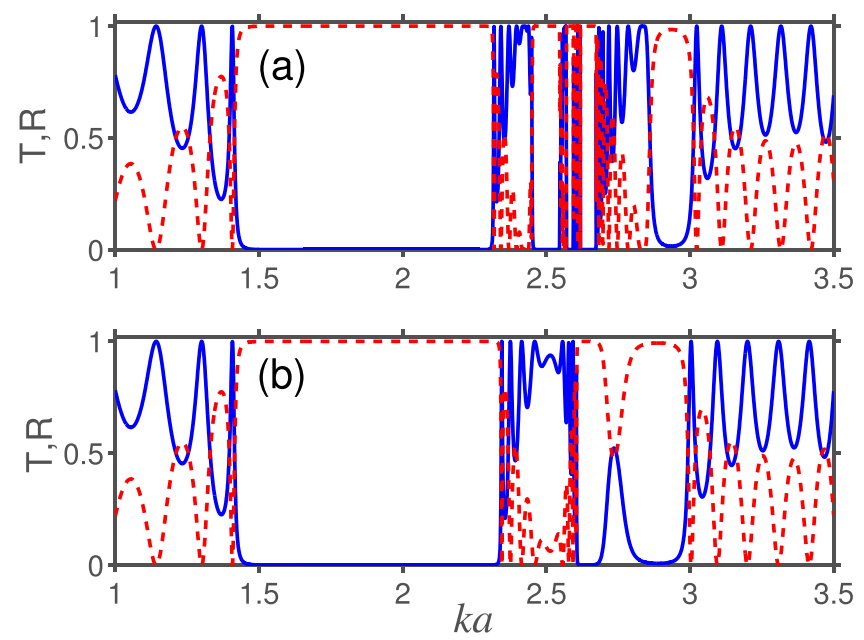

FIG. 6. Transmittance (solid blue lines) and reflectance (dashed red lines) for slab of $\mathrm{PhC}$ with rods made of a hypothetic lossless material with $\varepsilon=\operatorname{Re} \varepsilon_{P}, \varepsilon_{\infty}=10.9, \varepsilon_{0}=12.66$; (a) $\Gamma / \omega_{T}=2.5 \times 10^{-3}$ and (b) $\Gamma / \omega_{T}$ $=9.5 \times 10^{-2} ; k_{T} a=5 \pi / 6, d / a=0.4, N=8$, and $\theta=0$.

absorption maxima when nonzero $\operatorname{Im} \varepsilon_{P}$ is taken into account, compare to Fig. 4(a). Thus, the difference between Figs. 3(c) and $6(\mathrm{a})$ is only related to a very narrow $k a$-range, in which either $A>0$ if $\operatorname{Im} \varepsilon_{P} \neq 0$ or $A \equiv 0$ if $\operatorname{Im} \varepsilon_{P}=0$. Hence, there are such values of $\Gamma / \omega_{T}$ that behavior of $T$ and $R$ vs $k a$ and, in particular, number and location of the pass and stop bands is fully determined by $\varepsilon_{\infty}$ and $\operatorname{Re} \varepsilon_{P}$, except for the abovementioned $k a$-range, in which $\operatorname{Im} \varepsilon_{P}$ can be significant. The comparison of Figs. 3(c) and 6(a) indicates that $\operatorname{Re} \varepsilon_{P}$ may exert a rather strong effect on the spectra at small values of $\Gamma / \omega_{T}$.

However, the behavior observed in Fig. 3(e) at $k a>2.3$ has nothing to do with what is shown in Fig. 6(b). Hence, $T$ and $R$ can be strongly affected by $\operatorname{Im} \varepsilon_{P}$ within a wide $k a$ range, including location of the absorption, pass, and stop bands. Thus, two cases can be distinguished depending on whether their location is mainly affected by $\operatorname{Re} \varepsilon_{P}$ or by $\operatorname{Im} \varepsilon_{P}$. In other words, the difference may appear in whether the bands in case of the rods made of GaAs are inherited from the similar structures but in the zero-loss approximation.

Since a diffraction-free regime is considered here, it is reasonable to show whether periodicity is necessary, or one can use a homogeneous slab made of GaAs to obtain the same basic effects as those observed in Figs. 3 and 4. Let us compare this with the case of a homogeneous GaAs slab, whose thickness is close to that of the slab of PhC in Figs. 3, 4, 5(a), and 6 . The results for $T, R$, and $A$ are presented in Fig. 7. The observed features are similar to the slabs of $\mathrm{PhC}$ at the same values of $\Gamma / \omega_{T}$. For instance, at $\Gamma / \omega_{T}=2.5 \times 10^{-3}$, the strong absorption band is now wider, whereas switching between the regimes of $R \approx 1$ and $A \approx 1$ is not so sharp. It is interesting that, in contrast with Fig. 3(c), $A>0.9$ is observed in Fig. 7(a) at the upper edge of the polaritonic gap. The maximum is achieved at $k a=2.835$, where $\operatorname{Re} \varepsilon_{P} \approx 0.7$ and $\sqrt{\operatorname{Re} \varepsilon_{P}} k D / \pi \approx 6$ that corresponds to one of the Fabry-Perot transmission resonances.

At $\Gamma / \omega_{T}=6 \times 10^{-2}$, the stop band extends from $k a \approx$ 2.1 to $k a \approx 3.4$. It is now blueshift and a bit narrower than in
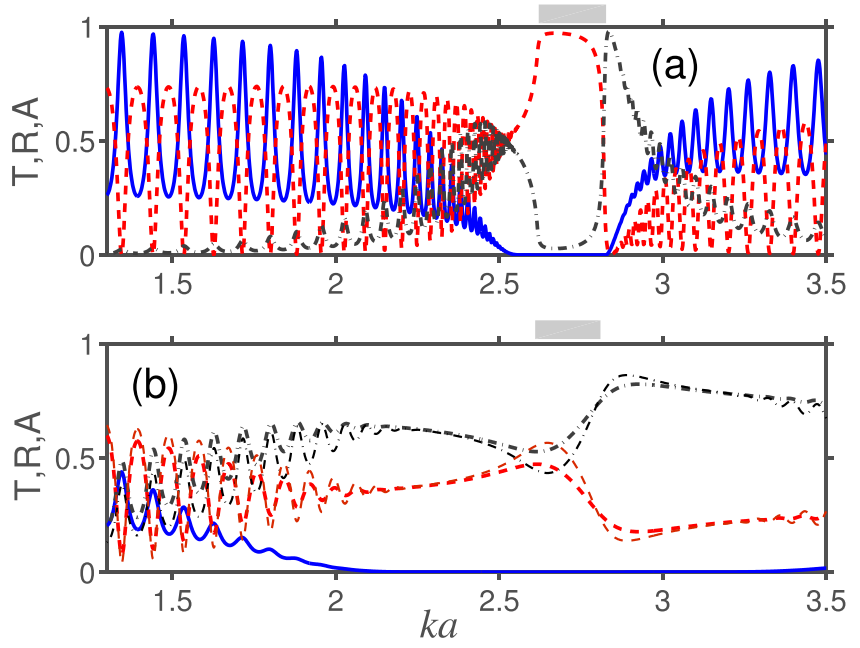

FIG. 7. Transmittance (solid blue lines), reflectance (dashed red lines), and absorption (dashed-dotted black lines) for a homogeneous slab with the same material characteristics as (a) in Fig. 3(c) and (b) in Fig. 3(e), thickness $D=8 a, \theta=0$.

Fig. 3(e), because there is no contribution of the photonic stop band in this case. However, in Fig. 7, these and other features are obtained at a larger volume occupied by a lossy material than in Figs. 3, 4, and 6. Indeed, the volume ratio is given by $\zeta=(2 a / d)^{2} / \pi$, i.e., $\zeta=7.96$ at $d / a=0.4$. Generally, a big portion of the above-mentioned effects in the slabs of $\mathrm{PhC}$ is inherited from the homogeneous slabs. It is important that the behavior of $T, R$, and $A$, which is connected with the specific properties of polar dielectric, may coexist in one $\mathrm{PhC}$ based structure with multiple photonic pass bands and stop bands, including those with the properties that cannot be obtained in homogeneous slabs of GaAs. Hence, the necessity of introducing a periodicity depends on whether multiple functions within different frequency ranges are required or not. However, locations of the maxima of $A$ with respect to the polaritonic gap can be different for the slab of PhC and homogeneous slab. This indicates the possible differences in the resulting mechanism and, at the same time, provides an additional degree of freedom. It is noteworthy that $\zeta>1$ might not be a necessary condition for obtaining strong absorption in the homogeneous slabs and is used here for comparison purposes.

\section{CONTINUOUS VARIATION OF $\Gamma$ AND $\theta$}

Next, we consider the behavior of $A$ at the simultaneous variation of $k a$ and $\Gamma / \omega_{T}$. The results are presented in Fig. 8 for $\theta=0,30^{\circ}$, and $60^{\circ}$. They confirm that the width and number of the absorption bands in the frequency domain can strongly depend on $\Gamma / \omega_{T}$. Among the observed features, one should notice two wide areas of high $A$, which are centered around $k a=2.4$ and $k a=2.8$ and one narrow area of high $A$ arising at $k a=2.55$. The former are extended from a nonzero value of $\Gamma / \omega_{T}<0.1$ and tend to merge at large values of $\Gamma / \omega_{T}$. This enables some tolerance regarding values of $\Gamma / \omega_{T}$ that can be used for an actual material. The lowerfrequency wide area of high $A$ corresponds to positive values of $\operatorname{Re} \varepsilon_{P}$, while the higher-frequency wide area of high $A$ may 

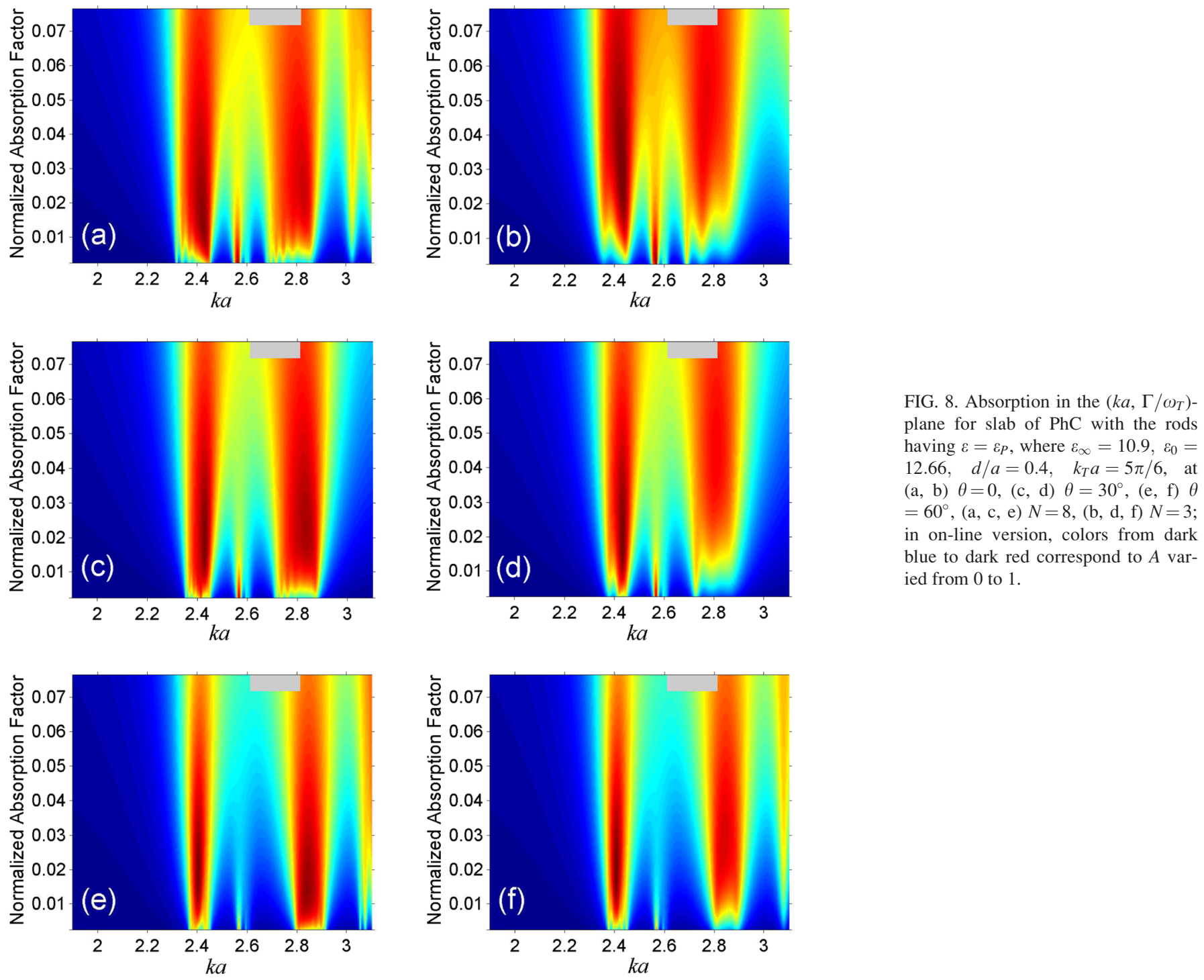

include the effects of negative, near-zero, and (at large $\Gamma / \omega_{T}$ ) positive values of $\operatorname{Re} \varepsilon_{P}$. The dark spots (dark red in on-line version), which correspond to the values of $A$ that are close to 1 , are noteworthy. Thus, there are ranges of optimal values of $\Gamma / \omega_{T}$, in which absorption is strongest for a given $k a$-value. Moreover, one can find such pairs of $\left(k a, \Gamma / \omega_{T}\right)$ that strong absorption appears within the dark areas for all three values of $\theta$. This allows one to expect the existence of wide $\theta$-ranges, in which $A \approx 1$. Comparing Fig. 8 with Figs. 1(d) and 1(e), one can see that only the narrow absorption band observed in Fig. 8 at $k a=2.55$ might remind one of the behavior types of $\varepsilon_{P}$ observed in Figs. 1(d) and 1(e). This band is mainly connected with the material properties, at least if $\Gamma / \omega_{T}<2 \times 10^{-2}$. In turn, the effect of geometry for the wide areas of high $A$ can be significant.

The comparison of Figs. 8(a) and 8(b), 8(c) and 8(d), and $8(\mathrm{e})$ and $8(\mathrm{f})$ shows that changing $N=8$ for $N=3$ slightly affects behavior of $A$. This feature argues in favor of the idea that the rod layers near the incidence interface can be the main contributors to the resulting absorption. It well coincides with Ref. 12, where a single layer of GaAs rods has been combined with a non-absorbing reflector. Accordingly, it may be expected that several upper rod layers might play the main role in absorption in the cases shown in Figs. 3-5. In the studied structures, the same rod layers can contribute to absorption directly and by enhancing absorption in the adjacent rod layer owing to reflections. However, clarifying the extent to which a certain rod layer contributes to the absorption is beyond the scope of this paper. Simultaneous contribution to absorption and reflection can be a reason why an increase of $N$ does not necessarily lead to larger values of $A$ at fixed $k a$ and $\Gamma / \omega_{T}$. At $N=3$, a bit higher $\Gamma$-threshold for the geometry related wide areas of high absorption is observed as compared to $N=8$. Narrowing the wide and narrow areas of high $A$ and weakening the extent of merging of the wide areas at large $\Gamma / \omega_{T}$ belong to the general effects arising when $\theta$ is increased. One should also mention the areas of weaker $A$ at $k a=3.1$, which either appear or disappear, depending on $\theta$ and $N$. The obtained results indicate that the effects observed in Figs. 3-5 at $\theta=0$ could also be wide-angle effects.

Now, we consider behavior of $A$ at a continuous variation of $\theta$. It is noteworthy that the possibility of obtaining a 

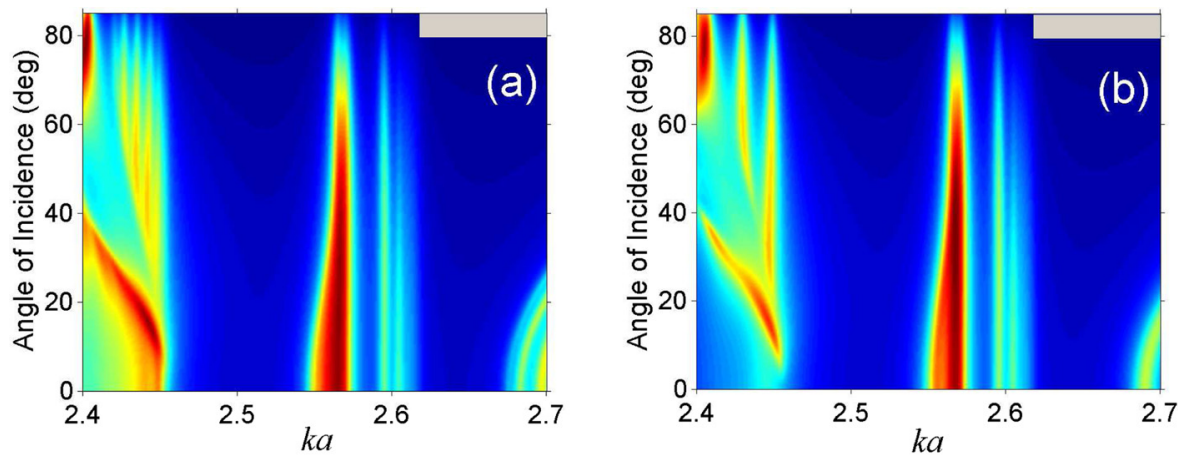

FIG. 9. Absorption in the $(k a, \theta)$-plane for slab of $\mathrm{PhC}$ with the rods having $\varepsilon=\varepsilon_{P}, \quad$ where $\quad \varepsilon_{\infty}=10.9, \quad \varepsilon_{0}=$ $12.66, d / a=0.4, k_{T} a=5 \pi / 6$, at $(\mathrm{a}, \mathrm{c})$ $N=8$, (b, d) $N=3$, (a, b) $\Gamma / \omega_{T}=2.5$ $\times 10^{-3}$, and (c, d) $\Gamma / \omega_{T}=6 \times 10^{-2}$;
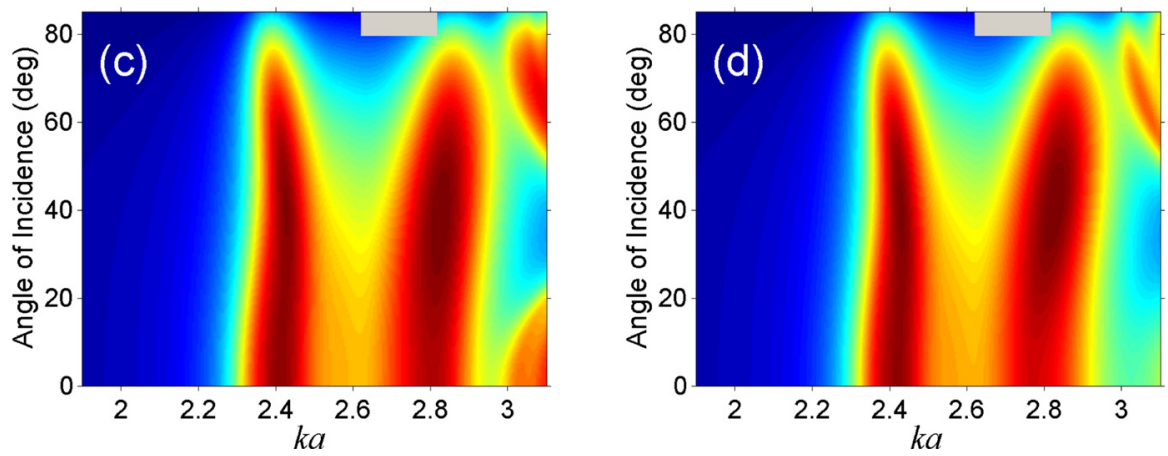
$\theta=0$; in on-line version, colors from dark blue to dark red correspond to $A$ varied from 0 to 1 .

weak dependence of $A$ on $\theta$ has recently been demonstrated for various absorbers. ${ }^{16-18}$ In Fig. 9, $A$ is shown in $(k a, \theta)$ plane for two typical values of $\Gamma / \omega_{T}$. One can see that $A>0.7$ remains in a wide range of $\theta$-variation. Hence, the absorption band can be tolerant regarding simultaneous variations in $k a, \theta$, and $\Gamma / \omega_{T}$ or just a variation in one of them. In particular, the observed behavior promises efficient absorption in case of nonmonochromatic incident waves with a wide frequency spectrum. The observed basic features qualitatively coincide with the predictions obtained by using the results from Fig. 8. At both $\Gamma / \omega_{T}=2.5 \times 10^{-3}$ and $\Gamma / \omega_{T}=6 \times 10^{-2}$, there are wide $\theta$-ranges, in which $A>0.9$. At $\Gamma / \omega_{T}=2.5 \times 10^{-3}$, one of such ranges occurs near $k a=2.55$ and, thus, corresponds to the narrow area of high $A$ in Fig. 8, i.e., it corresponds to large positive values of $\operatorname{Re} \varepsilon_{P}$. At $\Gamma / \omega_{T}=6 \times 10^{-2}$, two such ranges are located below and above the polaritonic gap. The areas of $A>0.9$ arising in the $(k a, \theta)$-plane should enable absorption of the beams with quite wide frequency and angular spectra. One should notice that two more low- $A$ bands at $\Gamma / \omega_{T}=2.5 \times 10^{-3}$ appear in the vicinity of the lower edge of the polaritonic gap. They correspond to the range of fast increase of $\operatorname{Re} \varepsilon_{P}$ but are located below $\max \operatorname{Re} \varepsilon_{P}$, see Fig. 1. In fact, the presence of three absorption bands between $k a=2.54$ and $k a=2.62$ is expected to be connected with splitting into many small pass bands separated by small band gaps that appear owing to the periodicity. ${ }^{6}$

From the comparison of Figs. 9(a) and 9(b) and Figs. 9(c) and 9(d), it is seen that the maps of $A$ at $N=8$ and $N=3$ are almost the same that confirms the main role of the upper rod layers. Here, this feature is demonstrated for a wide range of $\theta$ variation. Note that the results presented in Fig. 9 show different types of behavior of $A$ vs $\theta$ that may occur at a fixed $k a$.

To better illustrate the angular selectivity, Fig. 10 presents $A$ vs $\theta$ in the selected cases. In Figs. 10(a) and $10(\mathrm{~b})$, results are presented for the intermediate and small values of $\Gamma / \omega_{T}$, which lead, in our opinion, to the most interesting types of behavior of $A$. In Fig. 10(a), one of the cases corresponds to wide-angle absorption (for instance,
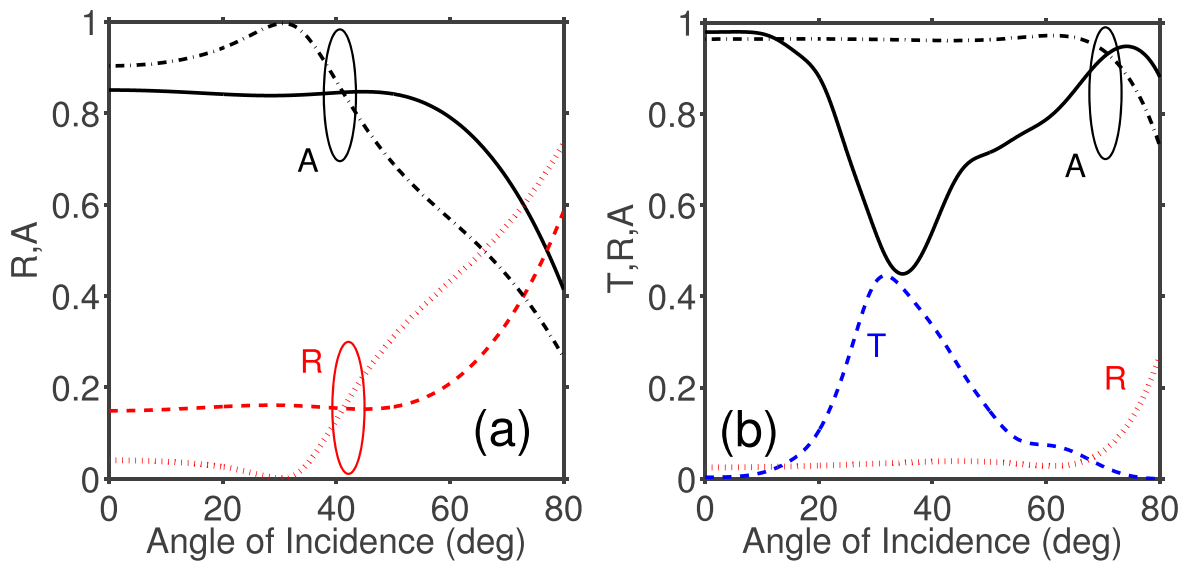

FIG. 10. Angular dependencies of $T$, $R$, and $A$ at fixed $k a$ : (a) solid black line- $A$, dashed red line $-R$ at $k_{T} a$ $=5 \pi / 6, \Gamma / \omega_{T}=6 \times 10^{-2}$ and $k a$ $=2.415$; dashed-dotted black line- $A$, dotted red line $-R$ at $k_{T} a=4 \pi / 6$, $\Gamma / \omega_{T}=2.5 \times 10^{-3}$ and $k a=2.018$; (b) solid black line- $A$, dashed blue line $-T$ at $k_{T} a=7 \pi / 12, \Gamma / \omega_{T}=2.5$ $\times 10^{-5}$ and $k a=1.816$; dashed-dotted black line $-A$, dotted red line $-R$ at $k_{T} a=7 \pi / 12, \Gamma / \omega_{T}=6 \times 10^{-5}$ and $k a=1.825 ; \quad \varepsilon_{\infty}=10.9, \varepsilon_{0}=12.66$, $d / a=0.4, N=8$. 
$A>0.8$ at $\theta<59^{\circ}$ when $\left.k a=2.415\right)$. The second one demonstrates the possible behavior of $A$ vs $\theta$, which can be considered as a Brewster-type behavior. In other words, $A=1$ is achieved at a nonzero $\theta$, with a further monotonous decrease of $A$, so that $A>0.9$ at $\theta<39^{\circ}$. In Fig. 10(b), we take very small values of $\Gamma / \omega_{T}$, which might be realizable with the aid of active impurities and, at the same time, enable important observations regarding the basic features in the absorption behavior. In the first case $(k a=1.816), A$ is close to 1 at small and large $\theta$, while a reflection-free regime with $A \approx$ $T \approx 0.5$ is realized at $\theta=32^{\circ}$. Here, transmission-mode band pass spatial (angular) filter is realized at intermediate $\theta$. In fact, this type of behavior of $A$ yields an alternative route to band pass spatial filtering. ${ }^{34,35}$ In the second case, an extremely wide band of nearly perfect absorption is obtained so that $A>0.95$ at $\theta<68^{\circ}$. Note that according to Eq. (1) for any pair of small but finite values of $\Gamma$ and $1-\omega / \omega_{T}$, there is a (very) narrow range of large $\operatorname{Re} \varepsilon_{P}$ and $\operatorname{Im} \varepsilon_{P}$. In such a range, behavior of $A$ could be similar to that observed in the second case in Fig. 10(b).

\section{DEFECT MODES}

As known, a very narrow pass band can be obtained in two ways: with the aid of structural defects that create defect modes $^{3}$ or by using strong narrowband variations in the material parameters. ${ }^{4}$ It has been shown above that an increase of $\Gamma$ can result in the suppression of narrow pass bands related to the narrowband variations in $\operatorname{Re} \varepsilon_{P}$ and in the appearance of a wide absorption band. Similar effects are expected to appear when the transmission spectrum contains the peaks connected with the structural defects. An example is presented in Fig. 11 for the slab of $\mathrm{PhC}$, which is distinguished from Figs. 3, 4, 8, and 9 in that the 3rd and 6th rod layers are removed. Besides, $k_{T} a$ is decreased in order to obtain good separation from the both stop band edges. If the rods are made of a dispersion-free material with $\varepsilon=\varepsilon_{\infty}$, two peaks of $T=1$ appear inside the lowest stop band of the corresponding defect-free structure, see Fig. 11(a). Adding the frequency dependent term to $\varepsilon_{P}$ at $\Gamma / \omega_{T}=2.5 \times 10^{-5}$ leads to redshift of the defect mode related peaks of $T=1$ [in Fig. 11(b), they are located near $k a=1.75]$ and in the appearance of new pass bands that are similar to those in Figs. 3(b), 3(c), and 4(a).

Comparing results for $\Gamma / \omega_{T}=2.5 \times 10^{-5}$ in Fig. 11(b) and $\Gamma / \omega_{T}=6 \times 10^{-2}$ in Fig. 11(c), one can see that the utilized variation in $\Gamma / \omega_{T}$ is sufficient for keeping just a weak reminiscence of defect mode related peaks in the spectrum of $T$. Suppression can be even stronger, for instance, if the 4th and 5th rod layers are removed, leading to a single wide centered defect. In this case, the peak of $T=1$ appears for $\Gamma / \omega_{T}=2.5 \times 10^{-5}$ (not shown) but is fully suppressed at $\Gamma / \omega_{T}=6 \times 10^{-2}$. At the same time, the spectra of $R$ and $A$ are expected to depend on that which rod layers actually contribute to the resulting absorption. In particular, if the line defects are located at a larger distance from the incidence interface than the contributing rod layers, the effect of defect modes on $T$ should not be significant. Moreover, reflections from the remaining layers can affect the dependences of $T$,
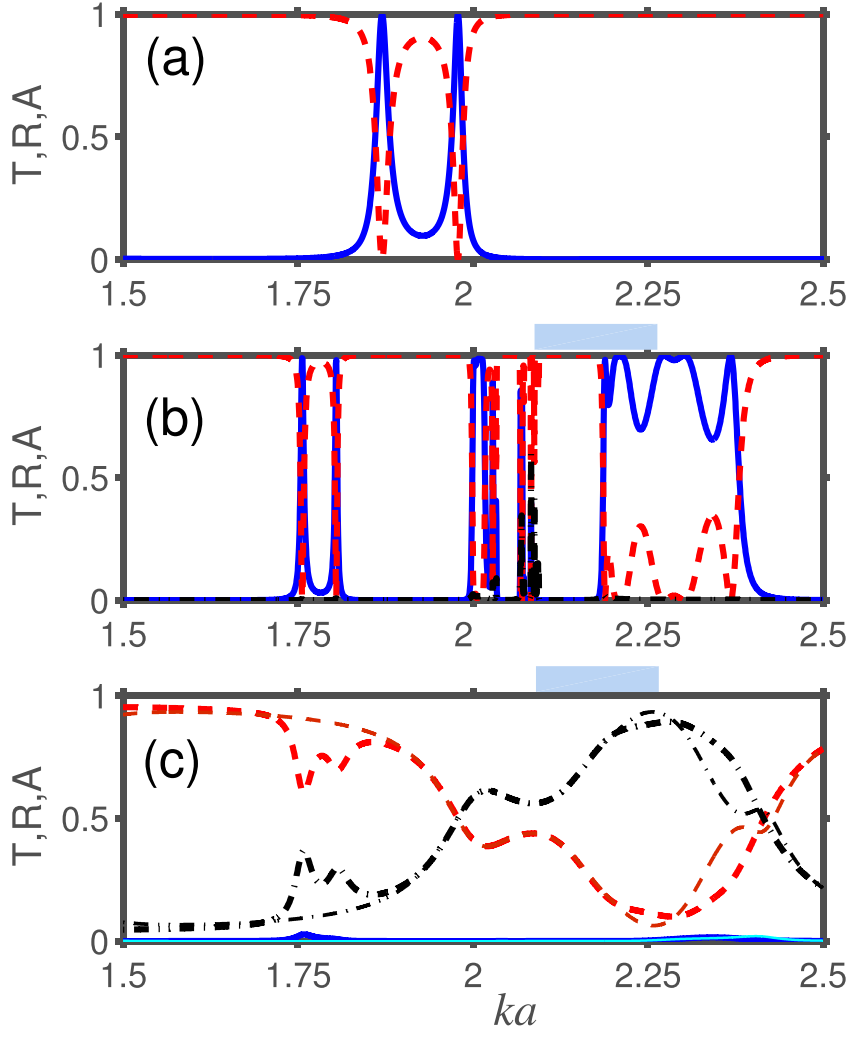

FIG. 11. $T$ (solid blue lines), $R$ (dashed red lines), and $A$ (dashed-dotted black lines) for slab of $\mathrm{PhC}$ with $N=8$, at $\varepsilon_{\infty}=10.9$, (a) $\varepsilon_{P}=\varepsilon_{\infty}$, (b, c) $\varepsilon_{P}$ given by (1) at $\varepsilon_{0}=12.66, k_{T} a=4 \pi / 6, d / a=0.4$, (b) $\Gamma / \omega_{T}=2.5 \times 10^{-5}$, (c) $\Gamma / \omega_{T}=6 \times 10^{-2} ; \theta=0 ; 3 \mathrm{rd}$ and 6 th rod layers are removed; in plot (c), $R$ and $A$ are shown by thinner lines in case when 4 th and 5 th rod layers are removed.

$R$, and $A$ on $k a$. However, in contrast with Ref. 12, one cannot unambiguously distinguish here between the layers that would mainly contribute to absorption or reflection. Indeed, all the rod layers are the same and each of them may play both roles simultaneously.

The above said is partially illustrated in Fig. 12 for the structures with a rather large number of the rod layers and two symmetrically placed line defects. The $k a$-range of $T \approx 0$ is very wide for both cases of $k_{T} a=4 \pi / 6$ and $k_{T} a=7 \pi / 12$, which correspond to the different locations of the polaritonic gap with respect to the edges of the periodicity related stop band. At $k_{T} a=7 \pi / 12$, the polaritonic gap covers the $k a$-range, in which the defect-mode related peaks of $T$ are observed in Fig. 11(a). The comparison of the results in Fig. 12 shows that there is just a weak difference in $\max A$ between the main structure with defects and the six-layer defect-free (uniform) slab of PhC. $\max A$ for the four-layer uniform slab is a bit smaller due to a weaker effect of reflections and/or a larger total volume of the absorbing material. For both structures with defects in Figs. 12(a) and 12(b), there is no signature of an effect that might be exerted by defect modes on transmission.

In order to better illustrate the role of the in-material losses at possible replacement of an initial structure, i.e., a slab of PhC with defects by a similar slab without defects and with a smaller number of the rod layers, we compare the results in Fig. 12 with those obtained at a smaller value of $\Gamma / \omega_{T}$. The latter are presented in Fig. 13. As expected, the 

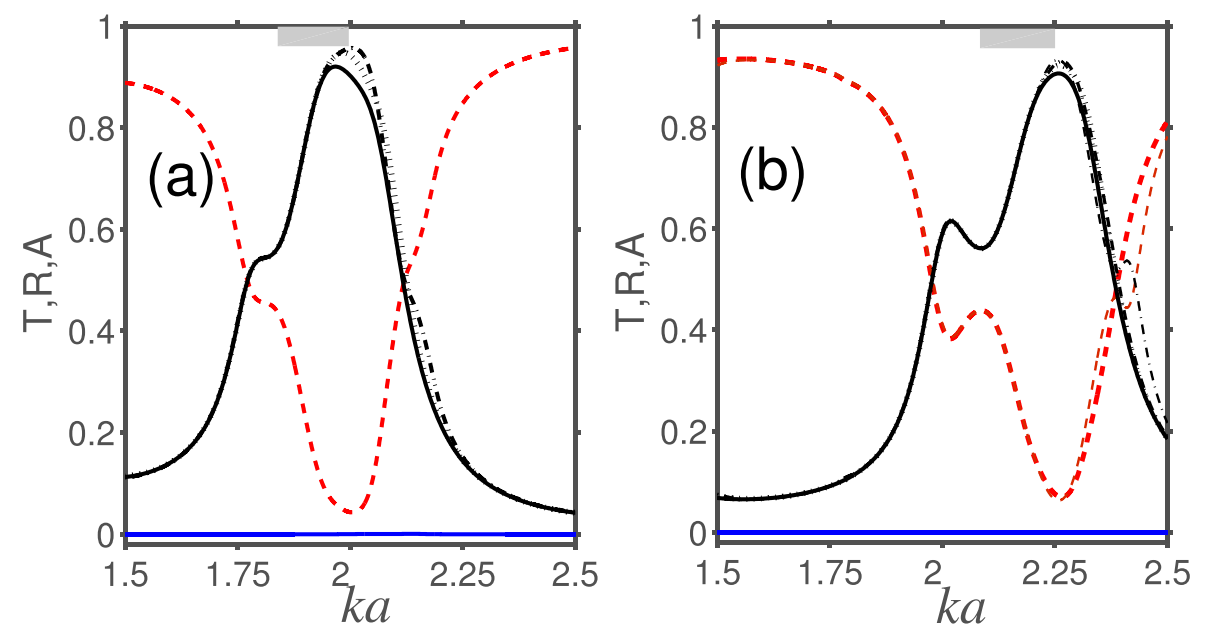

FIG. 12. $T$ (solid blue lines), $R$ (dashed red lines), and $A$ (dashed-dotted black lines) for slab of $\mathrm{PhC}$ at $\varepsilon_{\infty}=10.9$, $\varepsilon_{0}=12.66, \quad \Gamma / \omega_{T}=6 \times 10^{-2}, d / a$ $=0.4, \theta=0$, (a) $k_{T} a=7 \pi / 12, N=14$; (b) $k_{T} a=4 \pi / 6, N=14$ (thick lines) and $N=8$ (thin lines); 5th and 10th rod layers are removed for $N=14$ and 4 th and 5th rod layers do so for $N=8$; for comparison, $A$ is shown for defect-free slabs of the same $\mathrm{PhC}$ with $N=4$ (solid black line) and $N=6$ (dotted black line).
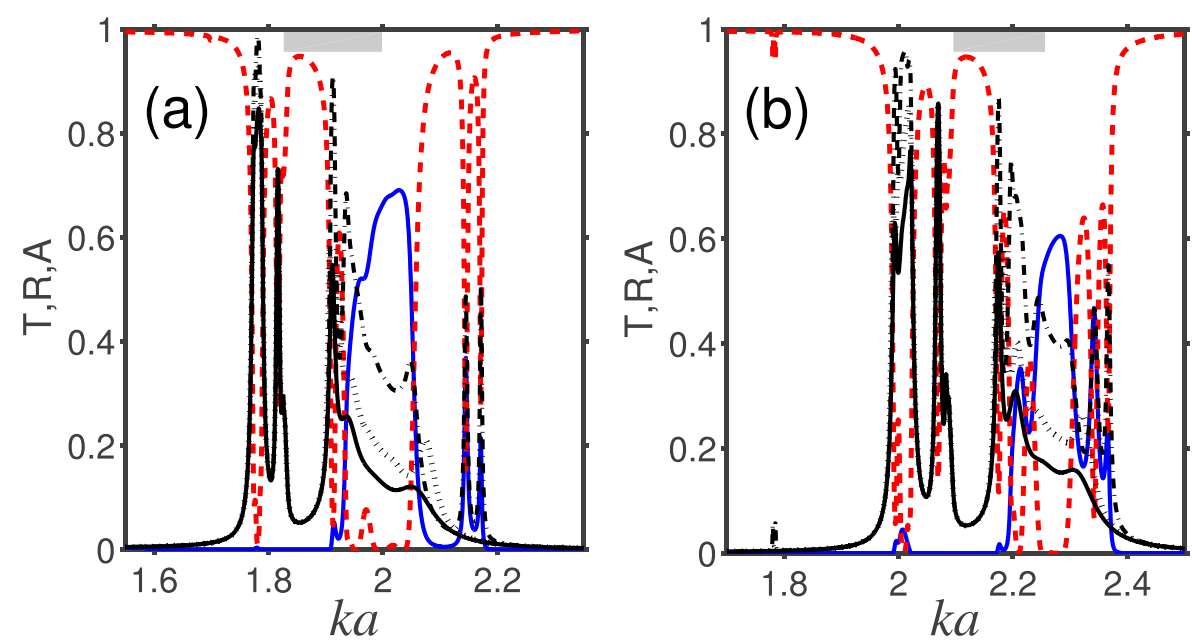

FIG. 13. $T$ (solid blue lines), $R$ (dashed red lines), and $A$ (dashed-dotted black lines) for slab of $\mathrm{PhC}$ at $\varepsilon_{\infty}=10.9$, $\varepsilon_{0}=12.66, \Gamma / \omega_{T}=2.5 \times 10^{-3}, d / a$ $=0.4, N=14, \theta=0$, (a) $k_{T} a=7 \pi / 12$, and (b) $k_{T} a=4 \pi / 6$; 5th and 10th rod layers are removed; for comparison, $A$ is shown for defect-free slabs of the same $\mathrm{PhC}$ with $N=4$ (solid black line) and $N=6$ (dotted black line).

differences between the compared structures are now stronger than in Fig. 12; they do not indicate an effect of defect modes, except for the vicinity of $k a=2.16$ in Fig. 13(a), where $A>0.4$ and $T>0.2$ at the maxima for the slab with defects but $A<5 \times 10^{-2}$ for the both uniform slabs. The
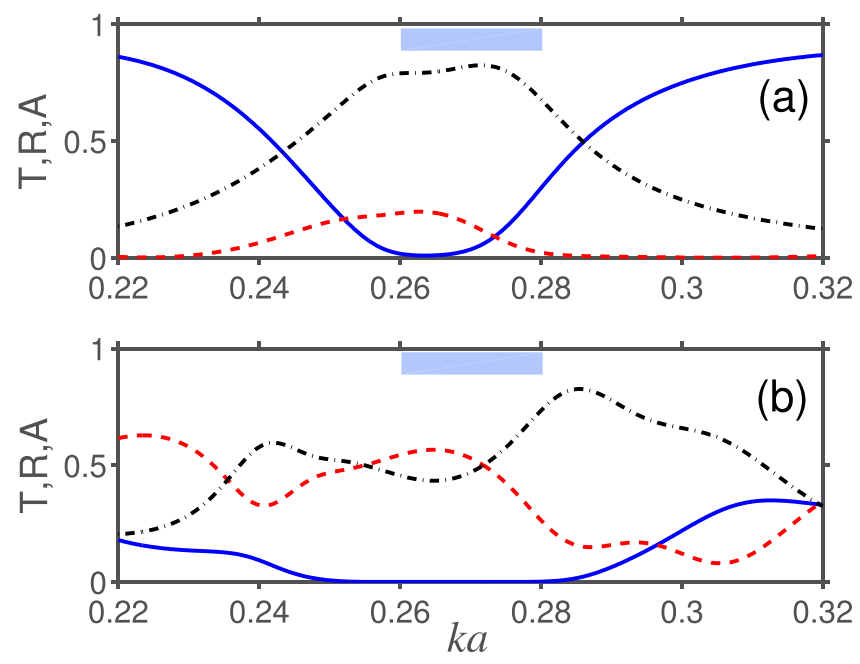

FIG. 14. $T$ (solid blue lines), $R$ (dashed red lines), and $A$ (dashed-dotted black lines) for (a) slab of PhC at $N=8$ and (b) homogeneous slab with $D=8 a ; \varepsilon_{\infty}=10.9, \varepsilon_{0}=12.66, k_{T} a=\pi / 12, \Gamma / \omega_{T}=6 \times 10^{-2}, d / a=0.4 ; \theta=0$. same remains true for the vicinity of $k a=2.36$ in Fig. 13(b), where $A>0.4$ and $T>0.2$ at the maxima for the slab with defects but $A<0.15$ for the both uniform slabs. Moreover, the shift of $k_{T} a$ in Fig. 13(b) with respect to Fig. 13(a) results in the appearance of a weak double peak of $A$ near $k a=1.8$, owing to the coupling of structural defects. Hence, some defect modes can be suppressed, whereas some new ones may appear at least if $\Gamma / \omega_{T}$ is not very large. Note that in Figs. 13(a) and 13(b), new defect-mode related peaks of $A$ and $T$ appear at $k a$-values that are well above the polaritonic gap and, thus, both $\operatorname{Re} \varepsilon_{P}$ and $\operatorname{Im} \varepsilon_{P}$ are not large.

\section{SUBWAVELENGTH BANDS}

Freedom in choice of $k_{T} a$ allows one to use such small values of $a$ that the polaritonic gap is located inside the lowest stop band as shown in Fig. 2(b). For instance, at $k_{T} a=\pi / 12$, not only a unit cell but also the entire slab composed of eight rod layers remains subwavelength and, hence, homogenization could be applicable (but not used here), see Ref. 8. Figure 14 presents the results for a slab of $\mathrm{PhC}$ and for a homogeneous GaAs slab having nearly the same thickness, i.e., $D=8 a$. Hence, the volume ratio $\zeta=7.96$ is kept. In spite of this, strong absorption is observed for both structures and expected to occur for thinner homogeneous slabs, too. For the slab of $\mathrm{PhC}$, the absorption band mainly 

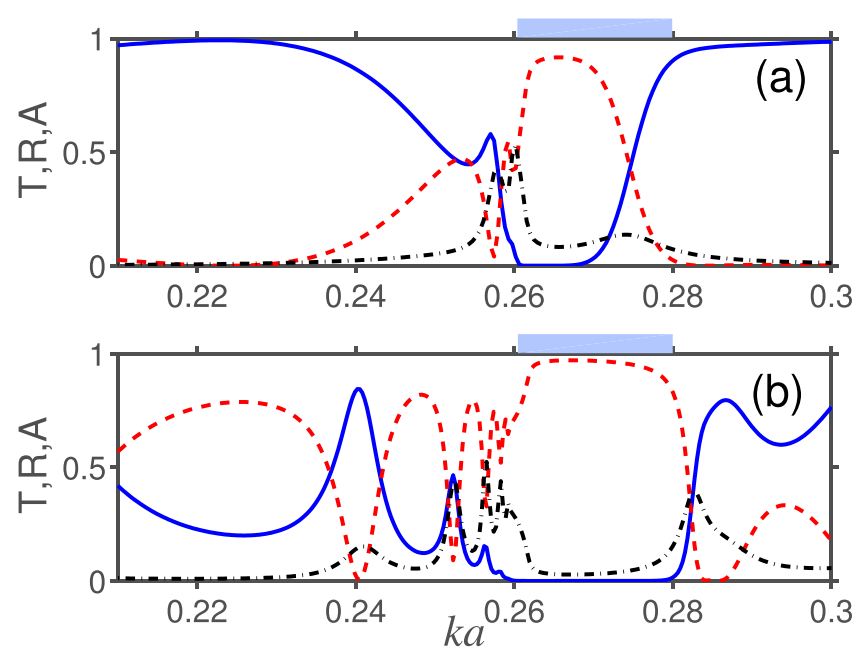

FIG. 15. Same as Fig. 14 but for $\Gamma / \omega_{T}=2.5 \times 10^{-3}$.

coincides with the polaritonic gap. In this case, we obtain $A>0.7$ at $0.254<k a<0.279$. For the homogeneous slab, a wider absorption band is obtained, while the strongest absorption occurs near and above the upper edge of the polariton gap. In particular, $A>0.7$ at $0.279<k a<0.294$. The strong difference between these two cases is mainly related to the behavior of $T$ and $R$ vs $k a$. In Fig. 14(a), one can see that $R>0$ only at $0.23<k a<0.28$. The ratio of $A / T$ is varied here in a wide range, including the case of $R=0$. For instance, $A \approx T \approx 0.45$ at $k a=0.243$ and $A \approx T \approx 0.5$ at $k a=0.286$. To compare, in Fig. 14(b), reflection is quite strong within the most part of the considered $k a$-range, while transmission tends to vanish at $0.25<k a<0.28$ and does not take such large values as in Fig. 14(a).

For the sake of completeness, Fig. 15 presents $A, R$, and $T$ vs $k a$ for the structures that differ from Fig. 14 in the value of $\Gamma / \omega_{T}$. Similarly to the above considered cases with larger $k_{T} a$, absorption is rather a narrowband effect. The strongest absorption achieved in Figs. 15(a) and 15(b) is nearly the same and, in turn, weaker than in Figs. 14(a) and 14(b). An absorption band is narrow and located at the lower edge of the polaritonic gap for the both structures. The second absorption band occurs for the homogeneous slab in Fig. 15 (b) at the upper edge of the polaritonic gap. The maximum is observed at $k a=0.283$, where $\operatorname{Re} \varepsilon_{P} \approx 0.45$ and $\sqrt{\operatorname{Re} \varepsilon_{P}} k D / \pi \approx 0.48$ so that we should be close to one of the Fabry-Perot reflection resonances. Generally, the main features of transmission and reflection in Fig. 15 do not differ from those in Fig. 14. It is noteworthy that in Fig. 15(a) we obtain $T>0.9$ at $0.21<k a<0.24$ and $k a>0.28$, i.e., narrow ranges of nonzero $A$ and $R$, which are related to the strong variations in $\varepsilon_{P}$, are embedded into a wide range of high transmittance.

\section{CONCLUSIONS}

To summarize, we studied the effect of the material absorption factor of GaAs varied in a very wide range on terahertz absorption, transmission, and reflection in slabs of a two-dimensional $\mathrm{PhC}$ based on the rods made of this material. As follows from the obtained results, a value of the absorption factor can be critical for obtaining a desired spectral behavior. Although some important features can be considered as those inherited from simpler structures, the periodic arrangement of the components, whose material is strongly absorptive within a limited frequency range, enables the full exploitation of multifunctionality advantages, because absorption bands in the vicinity of the polaritonic gap may coexist in one structure with different transmission and reflection regimes typical for $\mathrm{PhCs}$ which occur in different frequency ranges. At least two cases can be distinguished that result in narrow and wide absorption bands at small and large values of the absorption factor, respectively. Moreover, strong absorption can be obtained at a fixed frequency within a wide range of the incidence angle variation for both large and small values of the absorption factor. However, at the large values, absorption band is wide regarding variations of three different parameters: material absorption factor, frequency, and angle of incidence. This provides flexibility for design and for spectral and spatial characteristics of the incident waves. Besides, a specific angular dependence of absorption can be obtained in the transmission mode that leads to band pass spatial (angular) filtering.

Although new narrow pass and stop bands appear when the frequency dependent part of permittivity of a polar dielectric is taken into account, the former can be fully suppressed owing to an increase of the material absorption factor, as well as the defect mode transmission peaks arising in the vicinity of the polaritonic gap. This happens due to the main contribution of the upper rod layers to the resulting absorption. A more detailed study is required in order to clarify the roles of different rod layers. Indeed, all the layers in the studied structures are the same, so that each rod layer may contribute to the resulting absorption both directly and through reflections enhancing absorption in the rod layers that are located closer to the incidence interface.

It is expected that the obtained results might be qualitatively correct for other polar dielectrics, which differ from GaAs and each other in location and width of the polaritonic gap, but are described in the framework of the same model that reflects the same underlying physics. The difference in the properties of polar dielectrics gives us one more degree of freedom. Furthermore, two and more polar dielectrics could be utilized in one structure. One of the next steps will be connected with transferring the concept to much thinner structures. Although motivation for the use of impurities, either lossy (to extend an absorption band) or active (to compensate for the losses and obtain narrow absorption and transmission bands), has been explained, possible ways to realize such new materials are beyond the scope of this paper. However, the obtained results give the general concept and indicate the directions for further studies.

\section{ACKNOWLEDGMENTS}

This work was supported by the projects DPT-HAMIT, DPT-FOTON, and NATO SET-193, as well as by TUBITAK under the project Nos. 113E331, 109A015, and 109E301. The contribution of A.E.S. has partially been supported by the Matsumae International Foundation (Japan) 
and by TUBITAK in the framework of the visiting scientist programmes. E.O. acknowledges partial support from the Turkish Academy of Sciences.

${ }^{1}$ C. Kittel, Introduction to Solid State Physics (John Wiley and Sons, Hoboken, NJ, 2005).

${ }^{2}$ A. E. Serebryannikov, A. O. Cakmak, E. Colak, H. Caglayan, H. Kurt, and E. Ozbay, Opt. Express 22, 21806 (2014).

${ }^{3}$ K. Sakoda, Optical Properties of Photonic Crystals (Springer, Berlin, 2001).

${ }^{4}$ A. E. Serebryannikov and A. Lakhtakia, J. Opt. Soc. Am B 29, 328 (2012).

${ }^{5}$ S. Nojima, Phys. Rev. B 57, R2057 (1998).

${ }^{6}$ S. Nojima, Phys. Rev. B 59, 5662 (1999).

${ }^{7}$ M. V. Erementchouk, L. I. Deych, and A. A. Lisyansky, Phys. Rev. B 73, 115321 (2006).

${ }^{8}$ S. Foteinopoulou, M. Kafesaki, E. N. Economou, and C. M. Soukoulis, Phys. Rev. B 84, 035128 (2011).

${ }^{9}$ M. Kafesaki, A. A. Basharin, E. N. Economou, and C. M. Soukoulis, Photon. Nanostruct. Fund. Appl. 12, 376 (2014).

${ }^{10}$ A. E. Serebryannikov, A. O. Cakmak, and E. Ozbay, Opt. Express 20, 14980 (2012)

${ }^{11}$ C. Pfeiffer and A. Grbic, Phys. Rev. Lett. 110, 197401 (2013).

${ }^{12}$ A. E. Serebryannikov, S. Nojima, and E. Ozbay, Phys. Rev. B 90, 235126 (2014).

${ }^{13}$ N. I. Landy, S. Sajuyiqbe, J. J. Mock, D. R. Smith, and W. J. Padilla, Phys. Rev. Lett. 100, 207402 (2008).

${ }^{14}$ L. Huang, D. R. Chowdhury, S. Ramani, M. T. Reiten, S.-N. Luo, A. J. Taylor, and H.-T. Chen, Opt. Lett. 37, 154 (2012).

${ }^{15}$ S. Thongrattanasiri, F. H. L. Koppens, and F. J. Garcia de Abajo, Phys. Rev. Lett. 108, 047401 (2012).

${ }^{16}$ D. Liang, Y. Huo, Y. Kang, K. X. Wang, A. Gu, M. Tan, Z. Yu et al., Adv. Energy Mater. 2, 1254 (2012).
${ }^{17}$ C. Argyropoulos, K. Q. Le, N. Mattiucci, G. D'Aguanno, and A. Alu, Phys. Rev. B 87, 205112 (2013).

${ }^{18}$ M. J. Lockyear, A. P. Hibbins, J. R. Sambles, P. A. Hobson, and C. R. Lawrence, Appl. Phys. Lett. 94, 041913 (2009).

${ }^{19}$ Y. Wen, W. Ma, J. Bailey, G. Matmon, and X. Yu, IEEE Trans. Terahertz Sci. Technol. 5, 406 (2015).

${ }^{20}$ Y. Avitzour, Y. A. Urzhumov, and G. Shvets, Phys. Rev. B 79, 045131 (2009).

${ }^{21}$ M. A. Kats, R. Blanchard, P. Genevet, and F. Capasso, Nature Mater. 12, 20 (2013).

${ }^{22}$ K. B. Alici, F. Bilotti, L. Vegni, and E. Ozbay, J. Appl. Phys. 108, 083113 (2010).

${ }^{23}$ P. R. West, S. Ishii, G. V. Naik, N. K. Emani, V. M. Shalaev, and A. Boltasseva, Laser Photon. Rev. 4, 795 (2010).

${ }^{24}$ T. Magath and A. E. Serebryannikov, J. Opt. Soc. Am. A 22, 2405 (2005).

${ }^{25}$ M. M. Sigalas, C. M. Soukoulis, C. T. Chan, and K. M. Ho, Phys. Rev. B 49, 11080 (1994).

${ }^{26}$ H.-T. Chen, Opt. Express 20, 7165 (2012).

${ }^{27}$ R. Alaee, M. Farhat, C. Rockstuhl, and F. Lederer, Opt. Express 20, 28017 (2012).

${ }^{28}$ V. Kuzmiak, A. A. Maradudin, and A. R. McGurn, Phys. Rev. B 55, 4298 (1997).

${ }^{29}$ O. Toader and S. John, Phys. Rev. E 70, 046605 (2004).

${ }^{30}$ M. M. Sigalas, C. T. Chan, K. M. Ho, and C. M. Soukoulis, Phys. Rev. B 52, 11744 (1995).

${ }^{31}$ E. I. Smirnova, C. Chen, M. A. Shapiro, J. R. Sirigiri, and R. J. Temkin, J. Appl. Phys. 91, 960 (2002).

${ }^{32}$ A. E. Serebryannikov and T. Magath, Phys. Rev. A 76, 033828 (2007).

${ }^{33}$ A. Alu, M. G. Silveirinha, A. Salandrino, and N. Engheta, Phys. Rev. B 75, 155410 (2007).

${ }^{34}$ L. Maigyte and K. Staliunas, Appl. Phys. Rev. 2, 011102 (2015).

${ }^{35}$ E. Colak, A. O. Cakmak, A. E. Serebryannikov, and E. Ozbay, J. Appl. Phys. 108, 113106 (2010). 